\title{
Individual Cylinder Air-Fuel Ratio Estimation for a Gasoline Engine with Electromagnetic Valve Train
}

\author{
Yaxuan $\mathrm{Xu}$ and Siqin Chang \\ Department of Mechanical Engineering, Nanjing University of Science and Technology, Nanjing, China
}

\begin{abstract}
For the multi cylinder gasoline engine, the consistency of each cylinder is an important index to affect the emission and the power. In this paper, in order to reduce the air-fuel ratio $(\mathrm{A} / \mathrm{F})$ maldistribution of the engine based on the electromagnetic valve train (EMVT), an individual cylinder $\mathrm{A} / \mathrm{F}$ estimation algorithm is proposed for the individual cylinder $\mathrm{A} / \mathrm{F}$ control. Based on the analysis of the hybrid and transfer models of the exhaust of each cylinder in steady state, an individual A/F observer is established by using Kalman filter algorithm. Then the unknown parameters in the observer are identified by the differential evolution(DE) algorithm. Only a single wide area exhaust oxygen(UEGO) sensor is needed to identify the unknown parameters and estimate the $\mathrm{A} / \mathrm{F}$ of each cylinder. The combined simulation of GT-Power and Simulink validates the effectiveness of the proposed estimation approach. The results show that the proposed method can provide good estimation results under steady-state condition.
\end{abstract}

\section{Introduction}

$\mathrm{A} / \mathrm{F}$ is one of the most important control variables in the electronic control system of engine, and its control precision is closely related to engine power, economy and emission [1,2]. However, traditional A/F control methods can only regulate the $\mathrm{A} / \mathrm{F}$ at the confluence point. The $\mathrm{A} / \mathrm{F}$ imbalance of different cylinders in a multi-cylinder engine is unavoidable. There are many reasons for the $\mathrm{A} / \mathrm{F}$ maldistribution of each cylinder, and the most direct and main sources of $\mathrm{A} / \mathrm{F}$ deviations are the cylinder air charges maldistribution and the difference between cylinder injectors [3].

In order to eliminate the difference of $\mathrm{A} / \mathrm{F}$ between cylinders, the researchers put forward different control methods. Accurate measurement or estimation of individual $\mathrm{A} / \mathrm{F}$ is the key to the whole $\mathrm{A} / \mathrm{F}$ control strategy. A common method is to estimate the $\mathrm{A} / \mathrm{F}$ of each cylinder according to a single UEGO sensor signal placed at the confluence point of the exhaust manifold. According to the different treatment methods on the signal, there are two kinds of technical routes: Time domain method and frequency domain method. The time domain method is to establish the gas mixing process of each cylinder and the dynamic model of the sensor, and regulate the individual $\mathrm{A} / \mathrm{F}$ calculating from the mixture $\mathrm{A} / \mathrm{F}$. The frequency domain method is to extract the frequency components that coincide with the exhaust frequency in the UEGO sensor signal, and to eliminate the difference between cylinders' A/F by suppression of these spectral components [4-6]. Currently both time-domain modeling and frequency-domain modeling require installing an UEGO sensor at the exhaust outlet of each cylinder in the process of parameter identification, and have high consistency requirements for the response speed and delay characteristics of each sensor, increasing the cost and complexity of the test.

This paper focuses on the individual cylinder $\mathrm{A} / \mathrm{F}$ estimation based on the EMVT. In view of the imbalance of $\mathrm{A} / \mathrm{F}$ in four-cylinder gasoline engine with the EMVT, a method of estimating the individual cylinder $\mathrm{A} / \mathrm{F}$ in steady state condition is presented. In this paper, a time domain model is established to generate and transfer the $\mathrm{A} / \mathrm{F}$ of each cylinder, and a method of parameter identification using DE is proposed [7]. The identification of the model can be accomplished by using only one UEGO sensor at the manifold junction points to simplify the test process and reduce the cost. The Kalman Filter Observer is constructed by using the obtained parameters. The effectiveness of the identification and estimation methods is verified by the combination simulations of GT-Power and Simulink.

\section{System model}

\subsection{System Analysis}

The EMVT engine is modified on the basis of a $1.8 \mathrm{~L}$ four-cylinder port-injected gasoline engine with symmetrical exhaust manifolds, and a UEGO sensor is installed at the confluence of the exhaust manifold. The specification of the targeted engine is shown in Table I. Cylinder head of the engine was reprocessed to fit the installation of EMVT, as shown in Fig. 1. As a fully 
flexible engine valve train, the EMVT can adjust the air quality into each cylinder by adjusting the opening duration of the intake valve. In the EMVT developed by the task group, each valve is driven separately by the electromagnetic actuator, which can realize the full flexibility adjustment of valve lift, valve timing and valve movement [8-10]. The displacement curve of the performance test experiment of the electromagnetic actuated valve is shown in Fig. 2.

Table 1. Specification of the Engine

\begin{tabular}{|c|c|}
\hline Parameter & Value \\
\hline Displacement & $1.8 \mathrm{~L}$ \\
\hline Bore & $80 \mathrm{~mm}$ \\
\hline Stoke & $89 \mathrm{~mm}$ \\
\hline No. of cylinders & 4 \\
\hline Compression ratio & 10.5 \\
\hline Rated power & $95.7 \mathrm{~kW} @ 6000 \mathrm{rpm}$ \\
\hline Rated torque & $171.3 \mathrm{Nm} @ 4500 \mathrm{rpm}$ \\
\hline
\end{tabular}

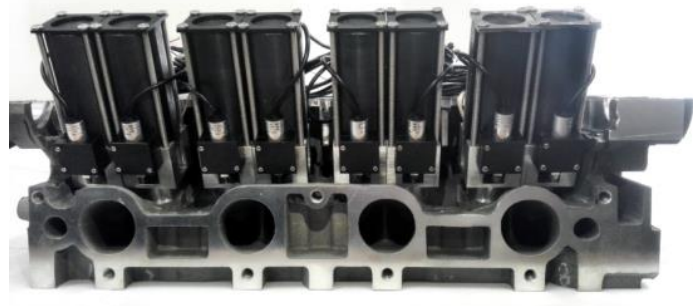

Figure 1. Test bench of engine cylinder head with EMVT
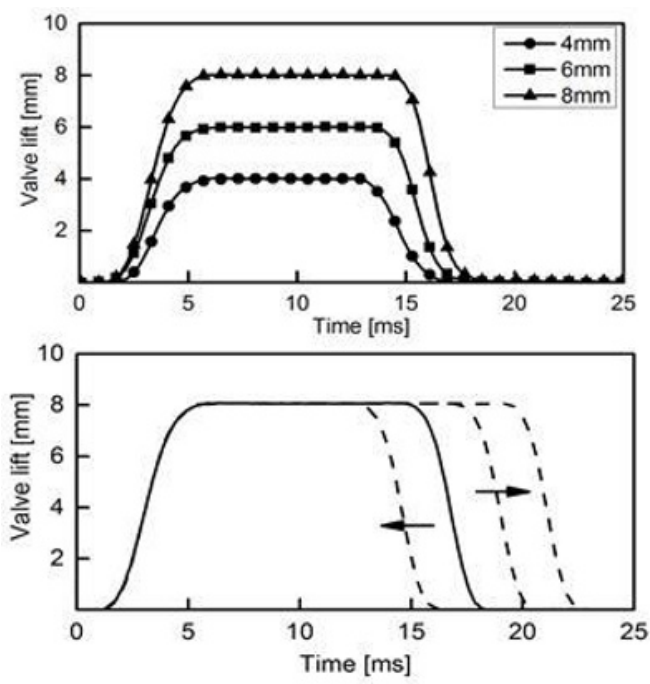

Figure 2. Test curve of valve lift and opening duration of EMVT.
In order to estimate the $\mathrm{A} / \mathrm{F}$ of each cylinder, an EMVT engine model, a mixture model of exhaust gas, a UEGO sensor model and uneven generation and transfer models need to be constructed. GT-power is used to build the one dimensional model of the prototype engine. Under steady state conditions, the oil film deposition and evaporation can be balanced, and the amount of oil in the cylinder is equal to the amount of injection so that the oilfilm model may be omitted in the steady-state control of the cylinder $\mathrm{A} / \mathrm{F}$.

\subsection{Exhaust Gas-Mixing Dynamics Model}

The gas mixture at the exhaust manifold confluence point at a certain time is formed by the exhaust gas from each cylinder. It can be introduced that the exhaust gas flow through the exhaust manifold changes with a simple periodic function under the constant speed condition. Our research object is a four-cylinder engine with a symmetrical exhaust manifold. Under the same working conditions, the proportional coefficients of the fixed rotation angles of each cycle are the same. We select the exhaust bottom dead centre (BDC) of each cylinder as the sampling point. The $\mathrm{A} / \mathrm{F}$ at the confluence point of exhaust manifold at instant $\mathrm{k}$ can be expressed as the following discrete equations:

$L_{m i x}(k)=\sum_{i=1}^{4} \mathrm{c}_{i} L_{c y l}(k-i+1)=C\left[\begin{array}{c}L_{c y l}(k) \\ L_{c y l}(k-1) \\ L_{c y l}(k-2) \\ L_{c y l}(k-3)\end{array}\right]$

$\boldsymbol{C}=\left[\begin{array}{llll}c_{1} & c_{2} & c_{3} & c_{4}\end{array}\right]$

Where $\mathrm{L}_{\text {mix }}(\mathrm{k})$ represents the $\mathrm{A} / \mathrm{F}$ of the gas at the confluence point of the exhaust manifold at instant $k$, and $\mathrm{L}_{\text {cyl }}(\mathrm{k})$ represents the $\mathrm{A} / \mathrm{F}$ of the gas at exhaust port of current exhausting cylinder. For the four-cylinder engine we have studied with a symmetrical exhaust manifold, the vector $\boldsymbol{C}$ corresponding to each exhaust BDC is basically the same.

\subsection{UEGO sensor model}

There is a transmission delay in the process of actual exhaust transmission to UEGO sensor installation point, and the sensor has dynamic effect. The UEGO sensor can be modeled as a first-order system with a propagation delay $T_{\text {lag }}$ and a time constant $\tau$ as shown in (3).

$$
G(\mathrm{~s})=\frac{1}{T_{\text {lag }} s+1} e^{-\tau s}
$$

\subsection{Uneven generation and transfer model}

In the case of identification parameters using a single sensor, the data available is only the initial intake valve 
closing time of each cylinder and the final $\mathrm{A} / \mathrm{F}$ of the UEGO sampling output. Therefore, in order to obtain the proportional coefficient matrix $\mathrm{C}$, it is necessary to analyze the whole process from the specified valve closing time to the $\mathrm{A} / \mathrm{F}$ of the mixing gas.

In the camless engine without throttle, the pressure in the intake manifold is very close to the atmospheric pressure, and the change rate of the gas pressure in the manifold is very small. Thus, the gas flow through the throttle valve is approximately the same as the gas flow through the intake port. From the engine charge efficiency and the ideal gas state equation, the air mass flow in the intake port of four-cylinder four-stroke gasoline engine can be obtained by

$$
\dot{m}_{\text {air }}=\frac{V_{s} \eta_{v} p_{i} n}{120 r T_{i}}
$$

Where Vs, $\eta v, r, T i, n$ are engine displacement, engine charge efficiency, gas constant, intake air temperature, and engine speed respectively. By integrating it in the intake stroke of the engine, the intake volume of each cylinder per cycle can be expressed as

$m_{a c}=\int_{i_{o}}^{i_{c}} \dot{m}_{a i r} d t$

Where $i_{o}, i_{c}$ are intake valve opening time and intake valve closing time respectively. Ideally, the intake air quality of each cylinder per cycle can be expressed as

$$
m_{a c}=\frac{\dot{m}_{a i r}\left[\frac{\mathrm{kg}}{h}\right]\left[\frac{h}{3600 s}\right]}{n\left[\frac{\mathrm{rev}}{\min }\right]\left[\frac{\mathrm{min}}{60 s}\right]\left[\left(n_{c y l} / 2\right) \text { stroke } \cdot \mathrm{rev}^{-1}\right]}=\frac{n_{c y l}}{30 n} \dot{m}_{\text {air }}
$$

Where $\mathrm{n}_{\text {cyl }}$ is the number of cylinders. However, due to differences in length and structure of the intake manifold, the actual intake air quality per cycle of each cylinder is not exactly the same. In order to accurately estimate the $\mathrm{A} / \mathrm{F}$ of each cylinder, the intake distribution deviation should be taken into account. The effect of deviation can be modelled as the cylinder effective coefficient $\mathrm{K}_{\mathrm{i}}$,

$m_{a c, i}=\bar{K}\left(1+K_{i}\right) \dot{m}_{a i r}\left(d_{u r}, n\right), \quad \mathrm{i}=1,2,3,4$

Where $\bar{K}$ is a constant coefficient, and $\mathrm{K}_{\mathrm{i}}$ is used to obtain the real air charge per stroke going into the $\mathrm{i}$-th cylinder from the air mass flow exiting the intake manifold. The $\dot{m}_{a i r, n}$ is related to the engine speed and intake valve opening duration. As shown in Fig. 3, the intake air mass flow of the engine under different intake valve opening durations is measured at the speed of 1600 $\mathrm{r} / \mathrm{min}$.

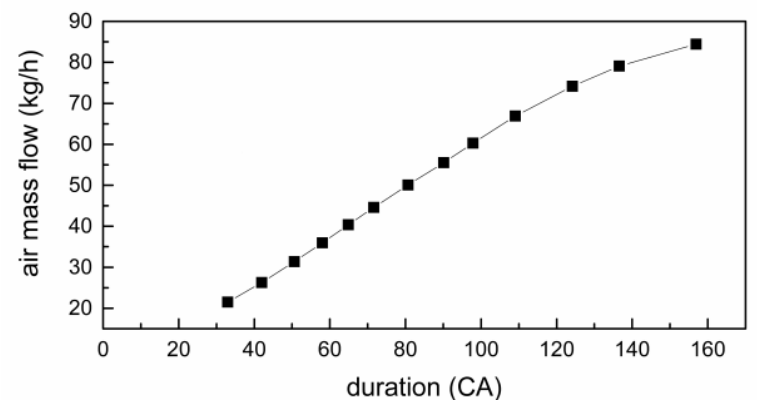

Figure 3. Air mass flow of different intake valve opening durations.

The fitting of cubic polynomial functions with the least squares method is used to fit the curve, and the fitting result is

$\dot{m}_{\text {air }, n=1600}=(-0.0000209) \mathrm{d}_{u r}{ }^{3}+0.00444 \mathrm{~d}_{u r}{ }^{2}+0.31 d_{u r}+7.0848$

Where $\dot{m}_{\text {air }, n}$ is the intake air flow measured in the intake manifold at a certain speed $n$ and $d_{u r}$ is the intake valve opening duration, where $\mathrm{d}_{\mathrm{ur}}=\mathrm{i}_{\mathrm{c}}-\mathrm{i}_{\mathrm{o}}$. The result of curve fitting is shown in Fig. 4. The result shows that the maximum relative error is less than $4 \%$.

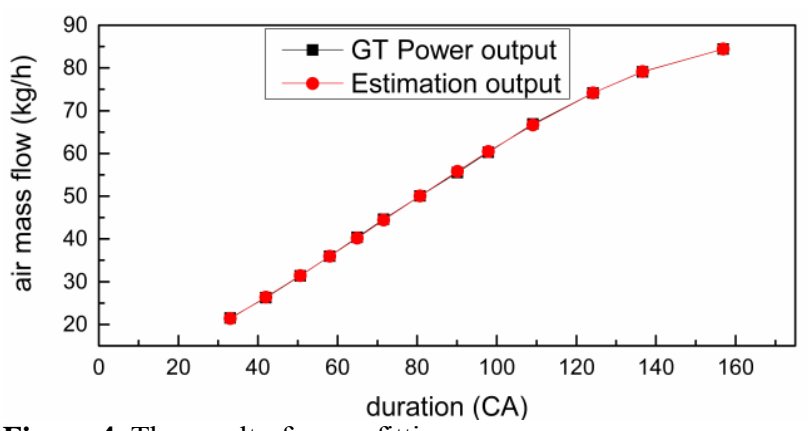

Figure 4. The result of curve fitting.

Then the intake air quality under a certain speed can be expressed as

$$
m_{a c, i}=\bar{K}\left(1+K_{i}\right) \dot{m}_{a i r, n}\left(d_{u r}\right), \quad \mathrm{i}=1,2,3,4
$$

Assuming that the cylinder injector is consistent, the fuel injection quantity of each cylinder can be expressed as

$m_{f}=G_{f s} t$

Where $G_{f s}$ is the average flow rate of each cylinder injector, and $t$ is the injection pulse width. Under steady conditions, the oil film effect is not taken into account, and the actual $\mathrm{A} / \mathrm{F}$ of each cylinder is

$$
L_{c y l, i}=m_{a c, i} / m_{f}
$$

Thus, the $\mathrm{A} / \mathrm{F}$ at the gas junction can be calculated according to the gas mixing time domain model described above. Finally, considering the characteristics of the UEGO sensor, the recursive form between the measurement value of the $\mathrm{A} / \mathrm{F}$ and the real $\mathrm{A} / \mathrm{F}$ can be 
obtained by discretization of the Tustin transform as below.

$$
L_{\text {sen }}(k)=\frac{2 T_{\text {lag }}-T_{s}}{2 T_{\text {lag }}+T_{s}} L_{s e n}(k-1)+\frac{T_{s}}{2 T_{\text {lag }}+T_{s}}\left[L_{\text {mix }}(k-d)-L_{\text {mix }}(k-d-1)\right]
$$

Where $\mathrm{L}_{\text {sen }}(\mathrm{k})$ represents the $\mathrm{A} / \mathrm{F}$ value measured by UEGO sensor in the manifold at instant $k, \mathrm{~T}_{\mathrm{s}}$ is the sampling period, and $\mathrm{d}$ is the number of delayed cycles, $\mathrm{d}=\tau / \mathrm{T}_{\mathrm{s}}$. As a result, the modelling of the transfer process from the intake valve opening duration of each cylinder to the measurement value of $\mathrm{A} / \mathrm{F}$ at the confluence point is completed.

\section{Individual cylinder A/F estimation and parameter identification}

\subsection{Establishment of Kalman filter Observer}

Kalman filter algorithm is a continuous "predictiveupdate" recursive calculation process, and it is efficient in real-time operation. The following is the basic equations of the discrete Kalman filter, in which the first two are the time-updating equations, and the latter three are the stateupdating equations [11].

$$
\left\{\begin{array}{l}
\hat{x}_{k}^{-}=A \hat{x}_{k-1}^{-} \\
P_{k}^{-}=A P_{k-1} A^{T}+Q \\
K_{k}=P_{k}^{-} C^{T}\left(C P_{k}^{-} C^{T}+R\right)^{-1} \\
\hat{x}_{k}=\hat{x}_{k}^{-}+K_{k}\left(Y_{k}-C \hat{x}_{k}^{-}\right) \\
P_{k}=\left(I-K_{k} C\right) P_{k}^{-}
\end{array}\right.
$$

Where, $\mathrm{Pk}$ is the estimation error; $\mathrm{Kk}$ is the kalman filter gain; $\mathrm{Q}$ and $\mathrm{R}$ are covariance matrices of system noise and measurement noise respectively. The Kalman filter estimates the current system state based on the previously calculated value and the current observational data. We take the $\mathrm{A} / \mathrm{F}$ of exhaust events' cylinders in the last cycle as the system state $\mathrm{X}$ :

$$
X(k)=\left[L_{c y l}(k), L_{c y l}(k-1), L_{c y l}(k-2), L_{c y l}(k-3)\right]^{T}
$$

And the mixing A/F measured by the UEGO sensor at the confluence point of the exhaust manifold is taken as the system observation $\mathrm{Y}$ :

$$
Y(k)=L_{\text {sen }}(k)
$$

The system state space model is established as follows:

$$
\left\{\begin{array}{l}
X(k+1)=A X(k)+W \\
Y(k+1)=C X(k+1)+V
\end{array}, \quad A=\left[\begin{array}{cccc}
0 & 0 & 0 & 1 \\
1 & 0 & 0 & 0 \\
0 & 1 & 0 & 0 \\
0 & 0 & 1 & 0
\end{array}\right]\right.
$$

Where, $\mathrm{A}$ is the state transition matrix of state space; $\mathrm{C}$ is the weighting factor matrix, $\mathrm{W}$ and $\mathrm{V}$ are system noise and measurement noise respectively.

\subsection{Parameter identification}

From the above model, we can see that in order to calculate the $\mathrm{A} / \mathrm{F}$ of each cylinder, we should find out the parameters of the proportional coefficient matrix $\mathrm{C}$. The parameters of the model are coupled with each other, and the differential evolution algorithm is used here for identification. In the case of a four-cylinder engine with a symmetrical exhaust manifold, the unknown parameters in the model are $\mathrm{K}_{1} \sim \mathrm{K}_{4}, \mathrm{c}_{1} \sim \mathrm{c}_{4}, \mathrm{~T}_{\text {lag }}$ and $\mathrm{d}$. It is possible to identify the UEGO parameters by a separate experiment so that the parameters to be identified can be further reduced to 8 . The intake valve closing time of each cylinder is specified in the Simulink, and the A/F of the mixing gas is output after the engine model running in the GT Power. Four different random sequences were used as the intake valve closing time of the four cylinders so that the $\mathrm{A} / \mathrm{F}$ of the mixing gas was evenly distributed within $\pm 10 \%$ of the equivalent ratio. The $\mathrm{A} / \mathrm{F}$ is sampled after the UEGO sensor at the confluence point of the exhaust manifold produces a stable periodic response. At the engine speed of $1600 \mathrm{r} / \mathrm{min}, 40$ sets of stable input and output are collected as identification data for parameter identification.

$\mathrm{DE}$ is for dealing with the continuous optimization problem. By using real number coding, an initial population $\left\{\boldsymbol{X}_{i, \boldsymbol{0}} \mid \mathrm{i}=1,2, \ldots, \mathrm{N}\right\}$ is randomly sampled from the feasible solution space. Real-valued vector $\boldsymbol{X}_{\boldsymbol{i}, \boldsymbol{G}}$ $=\left(\boldsymbol{X}_{i, \boldsymbol{G}}(1), \boldsymbol{X}_{i, \boldsymbol{G}}(2), \ldots, \boldsymbol{X}_{\boldsymbol{i}, \boldsymbol{G}}(\mathrm{D})\right)$ represent an individual in a population at generation $\mathrm{G}$. Where $\mathrm{D}$ is dimension of the objective problem, and $\mathrm{N}$ is the population size. Each individual in the population is a set of 8 parameters to be identified. The square sum of the error between the estimated output of $\mathrm{A} / \mathrm{F}$ and the actual measured output is calculated as the first fitness index of the individual.

$f(\boldsymbol{X})=100 \times \sqrt{\frac{\sum_{i=1}^{N}\left(L_{s e n, \mathrm{i}}-\hat{L}_{s e n, \mathrm{i}}(\boldsymbol{X})\right)^{2}}{N}}$

For each individual $\mathrm{X}_{\mathrm{i}}$ in the current population $\left\{\boldsymbol{X}_{\boldsymbol{i}, \boldsymbol{G}}\right.$ $\mathrm{i}=1,2, \ldots, \mathrm{N}\}, \quad \mathrm{DE}$ creates a mutant vector using "DE/rand/1" mutation strategies as shown in (18).

$V_{i, G}=X_{r 1, G}+F \cdot\left(X_{r 2, G}-X_{r 3, G}\right)$

Where $\mathrm{r} 1, \mathrm{r} 2$ and $\mathrm{r} 3$ are distinct integers randomly selected from the range $[1, \mathrm{~N}]$ and are also different form the index i. The scale factor $F$ is a positive control parameter for scaling the difference vector.

After mutation, DE performs a binomial crossover operator on $\boldsymbol{X}_{i, G}$ and $\boldsymbol{V}_{i, G}$ to generate a trial vector $\boldsymbol{U}_{i, G}$.

$\boldsymbol{U}_{i, G}(j)=\left\{\begin{array}{cc}\boldsymbol{V}_{i, G}(j), & \mathrm{R}_{j}(0,1) \leq P_{C R} \text { or } j=j_{\text {rand }} \\ \boldsymbol{X}_{i, G}(j), & \text { otherwise. }\end{array}\right.$ 
Where $\mathrm{j}=1,2, \ldots, \mathrm{D}, \mathrm{j}_{\text {rand }}$ is a randomly chosen integer in $[1, D], R_{j}(0,1)$ is a uniformly distributed random number between 0 and 1 which is generated for each $j$, and $\mathrm{P}_{\mathrm{CR}} \in[0,1]$ is called the crossover control parameter.

DE selects the better one from the trial vector $\boldsymbol{U}_{i, G}$ and the target vector $\boldsymbol{X}_{i, \boldsymbol{G}}$ to enter the next generation as shown in (20).

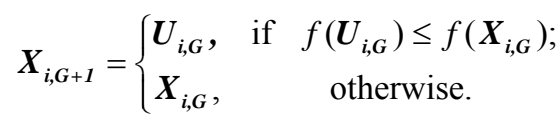

In order to test the accuracy of parameter identification with $\mathrm{DE}$ algorithm, the identification results are compared with those of the recursive least square (RLS) method. In RLS method, Different individual A/F $\mathrm{L}_{\text {cyl }}$ and the stable output of mixed $\mathrm{A} / \mathrm{F} \mathrm{L}_{\text {sen }}$ under the same conditions are taken as identification data for the parameter identification. Finally 4 sets of vector $C$ were obtained corresponding to 4 cylinders' exhaust BDC. The comparison of the parameter identification results is shown in the Table II, where \#1 \#4 represent cylinder1 cylinder4 respectively.

Table 2. Parameter identification results

\begin{tabular}{|c|c|c|c|c|c|}
\hline \multirow{2}{*}{ Parameters } & \multirow{2}{*}{ DE } & \multicolumn{4}{|c|}{ RLS } \\
\cline { 3 - 6 } & & $\# 1$ & $\# 2$ & $\# 3$ & $\# 4$ \\
\hline $\mathrm{C}_{1}$ & 0.516 & 0.535 & 0.543 & 0.563 & 0.537 \\
\hline $\mathrm{C}_{2}$ & 0.278 & 0.249 & 0.238 & 0.229 & 0.243 \\
\hline $\mathrm{C}_{3}$ & 0.132 & 0.148 & 0.151 & 0.146 & 0.152 \\
\hline $\mathrm{C}_{4}$ & 0.074 & 0.068 & 0.069 & 0.062 & 0.068 \\
\hline
\end{tabular}

It can be seen from the identification result of RLS that the parameters of four cylinders basically satisfy the symmetry hypothesis. Because of the accuracy of the model and the interference in the identification, there are some errors in the mixed coefficient identification, but it can also reflect the influence of the exhaust of each cylinder on the mixed air-fuel ratio.

\section{Estimation results of Kalman observer}

The estimation results of the individual $\mathrm{A} / \mathrm{F}$ are analyzed according to varied intake valve closing time as shown in Fig. 5. The intake valve opening time of each cylinder is 360 crankshaft angles, the cylinder fuel injection is constant, engine speed is $1600 \mathrm{r} / \mathrm{min}$, the average output torque is $100 \mathrm{Nm}$, and the lift of intake valve is $8 \mathrm{~mm}$. The IVCT of each cylinder changes as shown in Fig. 5(a). The mean zero white noise is added to the simulation to simulate the actual environment. As can be seen from Fig. 5 (b) $\sim$ (e), the large fluctuations of the $\mathrm{A} / \mathrm{F}$ at the transition point increased individual cylinder $\mathrm{A} / \mathrm{F}$ estimation error. After a short adjustment time, the error between the estimated value and the real value is stable within a certain range. The proposed Kalman Observer can suppress the system noise well, and since the maximum estimation error was less than $1 \%$ under steady-state conditions, the estimation performance was considered satisfactory.

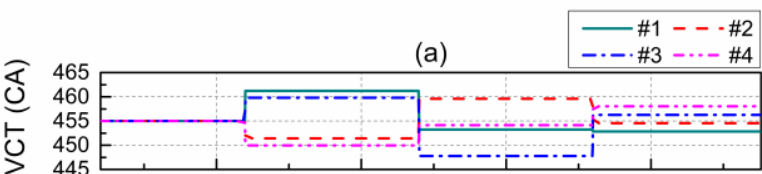

(b)

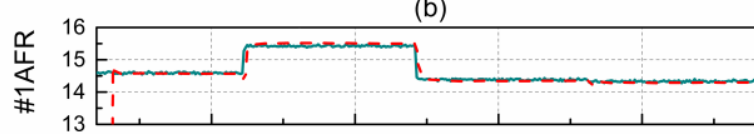

(c)

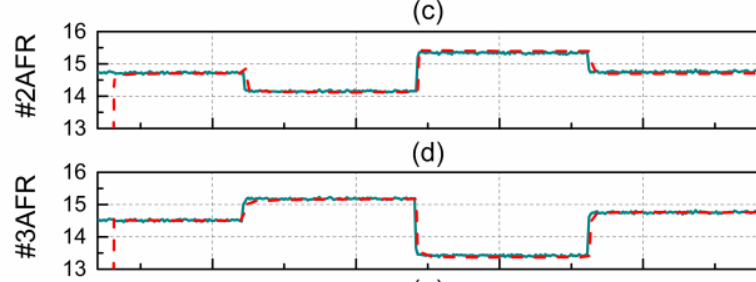

(e)

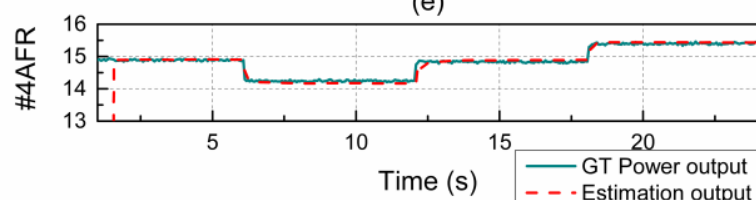

Figure 5. Individual cylinder A/F estimation results with respect to IVCT change (\#1 \#4 represent cylinder 1 cylinder4 respectively).

\section{Conclusion}

In this paper, an individual $\mathrm{A} / \mathrm{F}$ estimation approach for a four-cylinder gasoline engine with EMVT was developed for the maldistribution of the cylinder $\mathrm{A} / \mathrm{F}$. The main conclusions are as follows:

(1) The proposed Kalman filter observer allows estimation of the individual cylinder $\mathrm{A} / \mathrm{F}$ from a single UEGO sensor at the confluence point of the exhaust manifold. The observer is also applicable to $\mathrm{A} / \mathrm{F}$ estimates of other engines with symmetrical exhaust manifolds.

(2) The unknown parameters in the observer are identified by the differential evolution algorithm. Only a single UEGO sensor is used to complete the parameter identification, which simplifies the test and reduces the cost significantly compared with the common estimation methods.

(3) The proposed A/F observer can provide good estimation results under different valve opening duration, and has better ability to suppress the system noise, and the maximum error is less than $1 \%$ under steady-state condition.

\section{Acknowledgment}

This work was supported in part by the National Natural Science Foundation of China (grant number 51306090) and the Natural Science Foundation of Jiangsu Province, China (grant number BK20130762). 


\section{References}

1. $\mathrm{CW}$ Wu, RH Chen, JY Pu and TH lin, "The influence of air-fuel ratio on engine performance and pollutant emission of an SI engine using ethanol-gasolineblended fuels," Atmospheric Environment, vol. 38, pp. 7093-7100, ( 2004).

2. H Cho, K Min, SH Hwang and J Lee, "Prediction of the air-fuel ratio in transient conditions using a model of liquid fuel behaviour in the intake port of a sparkignition engine," Proceedings of the Institution of Mechanical Engineers Part D Journal of Automobile Engineering, vol. 214, pp. 731-740, (2000).

3. L Benvenuti, M. D. D Benedetto, S. D Gennaro and A. Sangiovanni-Vincentelli, "Individual cylinder characteristic estimation for a spark injection engine," Automatica, vol. 39 pp. 1157-1169, (2003).

4. J Kim, S Oh, K Lee, M Sunwoo, W Kim, C Lee and M Kim, "Individual Cylinder Air-Fuel Ratio Estimation Algorithm for Variable Valve Lift (VVL) Engines," Sae. Technical. Papers., 2010-01-0785, (2010).

5. T Hara, T Shen, Y Mutoh and Y Liu, "Periodic TimeVarying Observer-Based Learning Control of A/F Ratio in Multi-cylinder IC Engines," Springer International Publishing, pp. 65-83, (2017).
6. W Schick, C Onder, L Guzzella, "Individual cylinder air-fuel ratio control using Fourier analysis," IEEE Trans. Control Systems Technology, vol. 19, no. 5, pp. 1204-1213, (2011).

7. R. Mallipeddi, P.N. Suganthan, Q.K. Pan and M.F. Tasgetiren, "Differential evolution algorithm with ensemble of parameters and mutation strategies," Applied Soft Computing, vol. 11, no. 2, pp. 16791696, (2011).

8. J Xu, S Chang, X Fan and A Fan, "Effects of electromagnetic intake valve train on gasoline engine intake charging," Applied Thermal Engineering, vol. 96, pp. 708-715, (2016).

9. X Fan, S Chang, L Liu and J Lu, "Realization and optimization of high compression ratio engine with electromagnetic valve train," Applied Thermal Engineering, vol. 112, pp. 371-377, (2016).

10. L Liu and S Chang, "Improvement of valve seating performance of engine's electromagnetic valvetrain," Mechatronics, vol. 21, pp. 1234-1238, (2011).

11. Y Geng and J Wang, "Adaptive estimation of multiple fading factors in Kalman filter for navigation applications," Gps Solutions, vol. 12, pp. 273-279, (2007). 